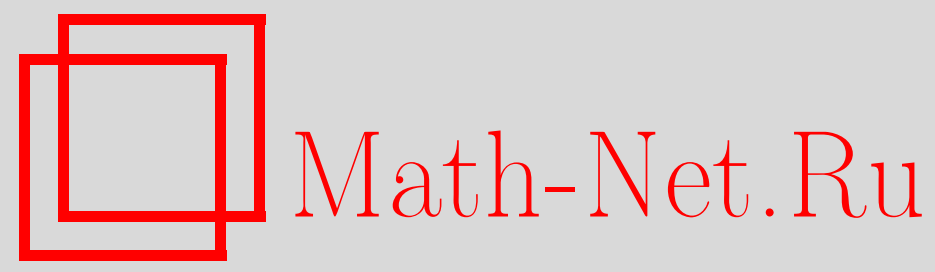

П. Н. Закревский, Вычисление коэффициента аддитивности для некоторых биюнктивных, слабо положительных и слабо отрицательных булевых функций, Матем. вопр. криптогр., 2015, том 6, выпуск $3,75-88$

DOI: https://doi.org/10.4213/mvk161

Использование Общероссийского математического портала Math-Net.Ru подразумевает, что вы прочитали и согласны с пользовательским соглашением http://www . mathnet.ru/rus/agreement

Параметры загрузки:

IP : 54.224 .187 .69

26 апреля 2023 г., 13:08:30

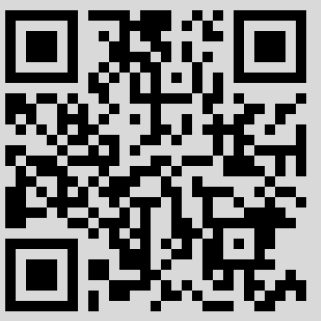


УДК: 512.57

\title{
Вычисление коэффициента аддитивности для некоторых биюнктивных, слабо положительных и слабо отрицательных булевых функций
}

\author{
П. Н. Закревский \\ Лаборатория ТВП, г. Москва
}

Получено 31.ХІІ.2014

Выводятся формулы для коэффициента аддитивности биюнктивных, слабо положительных или слабо отрицательных булевых функций специального вида. Приведено описание алгоритма вычисления коэффициента аддитивности произвольной булевой функции, заданной конъюктивной нормальной формой.

Ключевые слова: биюнктивные булевы функции, слабо положительные булевы функции, слабо отрицательные булевы функции, коэффициент аддитивности

Computation of the additivity coefficient of some bijunctive, weakly positive and weakly negative Boolean functions

P. N. Zakrevskiy

TVP Laboratory, Moscow

Abstract. We derive formulas for computation of the additivity coefficients of some bijunctive, weakly positive and weakly negative Boolean functions. An algorithm permitting to compute the additivity coefficient of any Boolean function given the normal conjunctive form is proposed.

Key words: bijunctive Boolean functions, weakly positive Boolean functions, weakly negative Boolean functions, additivity coefficient

Citation: Mathematical Aspects of Cryptography, 2015, vol. 6, no. 3, pp. 75-88 (Russian).

(C) 2015 П.Н. Закревский 


\section{Введение}

Работа посвящена изучению свойств нелинейности некоторых классов двоичных функций. В качестве меры нелинейности используется понятие коэффициента аддитивности функции.

Рассмотрим ситуацию, когда функция $f$ совпадает с одной линейной функцией на каком-либо подмножестве $M_{1}$ множества аргументов функции $f$ и с другой линейной функцией на подмножестве $M_{2}$, не пересекающемся с $M_{1}$. Если эти подмножества достаточно большие, то такое свойство функции является слабостью с криптографической точки зрения. В связи с этим представляется естественным метод выявления слабости булевой функции $f$, основанный на подсчете доли пар $(a, b), a, b \in V_{n}$, для которых выполнено свойство линейных функций $f(a+b)=f(a)+f(b)$.

Этот подход для анализа функций над кольцом $\mathbb{Z} / M$ и полем $G F\left(p^{k}\right)$ применялся ранее А. С. Амбросимовым.

В настоящей статье рассматриваются способы вычисления коэффициента аддитивности, введенного в [2], для некоторых подклассов классов биюнктивных, слабо положительных и слабо отрицательных булевых функций. Сначала приводятся определения и свойства двоичных функций, используемые в доказательствах основных результатов. Далее выводятся формулы для коэффициента аддитивности в случае, когда функции представимы в виде произведения функций от непересекающихся наборов переменных. В заключительной части работы приводится описание алгоритма вычисления коэффициента аддитивности произвольной булевой функции, заданной конъюнктивной нормальной формой.

\section{1. Основные определения и свойства}

Введем необходимые обозначения и определения:

$\overline{m, n}=\{m, m+1, \ldots, n\}$, где $m, n \in \mathbb{Z}$ и $m<n ;$

$V_{n}-$ множество двоичных векторов размерности $n$;

$\theta$ - нулевой вектор;

$\alpha=\left(a_{1}, \ldots, a_{n}\right), a_{i} \in\{0,1\}, i \in \overline{1, n},-$ двоичный вектор размерности $n$;

$\|\alpha\|=\sum_{i=1}^{n} a_{i}-$ вес вектора $\alpha$;

$F_{n}$ - множество всех булевых функций от $n$ переменных;

$\|f\|=\left|\left\{\alpha \in V_{n}: f(\alpha) \neq 0\right\}\right|-$ вес функции $f \in F_{n}$.

Пользуясь определениями, введенными в [5], опишем классы изучаемых функций. 
Булева функция $f\left(x_{1}, \ldots, x_{n}\right)$ называется биюнктивной, если существует ее представление в виде следующей конъюнктивной нормальной формы (КНФ):

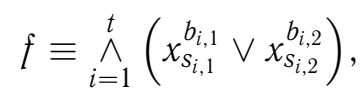

где $b_{i, j} \in\{0,1\}, s_{i, j} \in \overline{1, n}, i=\overline{1, t}, j=1,2, x^{0}=x, x^{1}=\bar{x}$.

Булева функция $f\left(x_{1}, \ldots, x_{n}\right)$ называется слабо положительной, если существует ее представление в виде КНФ:

$$
f \equiv \bigwedge_{i=1}^{t}\left(x_{s_{i, 1}}^{b_{i, 1}} \vee x_{s_{i, 2}} \vee \ldots \vee x_{s_{i, n_{i}}}\right)
$$

где $b_{i, 1} \in\{0,1\}, i=\overline{1, t}$.

Булева функция $f\left(x_{1}, \ldots, x_{n}\right)$ называется слабо отрицательной, если существует ее представление в виде

$$
f \equiv \bigwedge_{i=1}^{t}\left(x_{s_{i, 1}}^{b_{i, 1}} \vee \bar{x}_{s_{i, 2}} \vee \ldots \vee \bar{x}_{s_{i, n_{i}}}\right)
$$

где $b_{i, 1} \in\{0,1\}, i=\overline{1, t}$.

С основными результатами и свойствами рассматриваемых классов функций можно ознакомиться в [3], [4].

Перейдем к рассмотрению специальных параметров функции. Для произвольной булевой функции $f\left(x_{1}, \ldots, x_{n}\right)$ и набора $\alpha \in V_{n}$ определим параметр $\Delta_{\alpha}^{f}$ формулой (см., например, [2])

$$
\Delta_{\alpha}^{f}=2^{n-1}-\|f(x) \oplus\langle\alpha, x\rangle\|
$$

где $\alpha=\left(a_{1}, \ldots, a_{n}\right) \in V_{n}, x=\left(x_{1}, \ldots, x_{n}\right) \in V_{n}$ и $\langle\alpha, x\rangle=a_{1} x_{1} \oplus \ldots \oplus a_{n} x_{n}$.

Рассмотрим выражение

$$
\operatorname{Ad}(f)=\frac{\left|\left\{(\alpha, \beta) \in V_{n}^{2}: f(\alpha \oplus \beta)=f(\alpha) \oplus f(\beta)\right\}\right|}{\left|V_{n}\right|^{2}},
$$

где $\alpha=\left(a_{1}, \ldots, a_{n}\right) \in V_{n}, \beta=\left(b_{1}, \ldots, b_{n}\right) \in V_{n}, f \in F_{n}$. Величину $\operatorname{Ad}(f)$ будем называть коэффициентом аддитивности двоичной функции $f$. В [1] описаны основные свойства коэффициента $\operatorname{Ad}(f)$ и способы его вычисления. Один из результатов устанавливает связь между коэффициентами $\Delta_{\alpha}^{f}$ функции и величиной коэффициента аддитивности:

$$
\operatorname{Ad}(f)=\frac{1}{2}+\frac{1}{2^{3 n-2}} \sum_{\alpha \in V_{n}}\left(\Delta_{\alpha}^{f}\right)^{3} .
$$


Далее при вычислении величины $\mathrm{Ad}(f)$ для функций из рассматриваемых классов будем использовать формулу (2).

Приведем основные свойства коэффициентов $\Delta_{\alpha}^{f}$, следуя работе [2].

Свойство 1.1. Если двоичная функция $f\left(x_{1}, \ldots, x_{n}\right) \in F_{n}$ несущественно зависит от переменных $x_{k+1}, \ldots, x_{n}$ :

$$
f\left(x_{1}, \ldots, x_{n}\right)=g\left(x_{1}, \ldots, x_{k}\right),
$$

mo

$$
\Delta_{\left(a_{1}, \ldots, a_{n}\right)}^{f}= \begin{cases}2^{n-k} \Delta_{\left(a_{1}, \ldots, a_{k}\right)}^{g}, & \text { если } a_{k+1}=\ldots=a_{n}=0 \\ 0 & \text { в противном случае }\end{cases}
$$

Свойство 1.2. Если двоччная функция $f\left(x_{1}, \ldots, x_{n}\right)$ представляется в виде суммы двоичных функций от непересекающихся наборов переменных:

$$
f\left(x_{1}, \ldots, x_{n}\right)=f_{1}\left(x_{1}, \ldots, x_{k}\right) \oplus f_{2}\left(x_{k+1}, \ldots, x_{n}\right) \text {, }
$$

mо $\Delta_{\alpha}^{f}=2 \Delta_{\alpha_{1}}^{f_{1}} \Delta_{\alpha_{2}}^{f_{2}}$, где $\alpha=\left(a_{1}, \ldots, a_{n}\right), \alpha_{1}=\left(a_{1}, \ldots, a_{k}\right)$, $\alpha_{2}=\left(a_{k+1}, \ldots, a_{n}\right)$ и $a_{i} \in\{0,1\}$ для каждого $i \in \overline{1, n}$.

Воспользуемся приведенными свойствами для нахождения коэффициентов $\Delta_{\alpha}^{f}$ функции $f$, представленной в виде конъюнкции двух функций от непересекающихся наборов переменных.

Утверждение 1.1. Пусть двоичная функция $f\left(x_{1}, \ldots, x_{n}\right)$ представима в виде конъюнкции функций $f_{1}\left(x_{1}, \ldots, x_{n_{1}}\right) \wedge f_{2}\left(x_{n_{1}+1}, \ldots, x_{n_{1}+n_{2}}\right)$ от непересекающихся наборов переменных, и $n_{1}+n_{2} \leq n$. Тогда

$$
\Delta_{\alpha}^{f}=\left\{\begin{array}{c}
2^{n-1}-2^{n-n_{1}-n_{2}}\left(2^{n_{1}-1}-\Delta_{\theta}^{f_{1}}\right)\left(2^{n_{2}-1}-\Delta_{\theta}^{f_{2}}\right), \text { если } \alpha=\theta, \\
2^{n-n_{i}-1} \Delta_{\alpha_{i}}^{f_{i}}-2^{n-n_{1}-n_{2}} \Delta_{\alpha_{1}}^{f_{1}} \Delta_{\alpha_{2}}^{f_{2}}, \text { если } \alpha_{i} \neq \theta, \alpha_{j}=\theta \\
(i, j \in\{1,2\}, i \neq j), \alpha_{0}=\theta, \\
-2^{n-n_{1}-n_{2}} \Delta_{\alpha_{1}}^{f_{1}} \Delta_{\alpha_{2}}^{f_{2}}, \text { если } \alpha_{1} \neq \theta, \alpha_{2} \neq \theta, \alpha_{0}=\theta, \\
0, \text { если } \alpha_{0} \neq \theta
\end{array}\right.
$$

где $\alpha=\left(a_{1}, \ldots, a_{n}\right) \in V_{n}, \alpha_{1}=\left(a_{1}, \ldots, a_{n_{1}}\right) \in V_{n_{1}}, \alpha_{2}=\left(a_{n_{1}+1}, \ldots, a_{n_{1}+n_{2}}\right) \in V_{n_{2}}$, $\alpha_{0}=\left(a_{n_{1}+n_{2}+1}, \ldots, a_{n}\right) \in V_{n-n_{1}-n_{2}}$.

Доказательство. Рассмотрим сумму двух двоичных функций $f_{1}\left(x_{1}, \ldots, x_{n_{1}}\right) \oplus f_{2}\left(x_{n_{1}+1}, \ldots, x_{n_{1}+n_{2}}\right)$. Вычислим значение коэффициента $\Delta_{\alpha}^{f_{1} \oplus f_{2}}$. Для этого воспользуемся представлением (1) и известным свойством двоичных векторов

$$
\forall \alpha, \beta \in V_{n}\|\alpha \oplus \beta\|=\|\alpha\|+\|\beta\|-2\|\alpha \times \beta\|,
$$


где $\alpha \times \beta=\left(a_{1} \wedge b_{1}, \ldots, a_{n} \wedge b_{n}\right)$. Получим:

$$
\Delta_{\alpha}^{f_{1} \oplus f_{2}}=\left\{\begin{array}{r}
\Delta_{\alpha}^{f_{1}}+\Delta_{\alpha}^{f_{2}}-2 \Delta_{\alpha}^{f_{1} \wedge f_{2}}, \text { если } \alpha \neq \theta, \\
2^{n-1}+\left(\Delta_{\alpha}^{f_{1}}+\Delta_{\alpha}^{f_{2}}-2 \Delta_{\alpha}^{f_{1} \wedge f_{2}}\right), \text { если } \alpha=\theta .
\end{array}\right.
$$

Так как наборы существенных переменных функций $f_{1}$ и $f_{2}$ не пересекаются, то, используя свойства $1.1,1.2$, находим искомые выражения для коэффициентов $\Delta_{\alpha}^{f_{1} \wedge f_{2}}$.

Применяя $t-1$ раз утверждение 1.1 , можно вычислить значения коэффициентов $\Delta_{\alpha}^{f}$ функции, представленной в виде конъюнкции $t$ функций:

$$
f\left(x_{1}, \ldots, x_{n}\right)=\bigwedge_{i=1}^{t} f_{i}\left(x_{n_{0}+\ldots+n_{i-1}+1}, \ldots, x_{n_{0}+\ldots+n_{i}}\right), n_{0}=0
$$

каждая из которых существенно зависит от $n_{i}$ переменных, $i=\overline{1, t}$, а наборы $\left(x_{n_{0}+\ldots+n_{i-1}+1}, \ldots, x_{n_{0}+\ldots+n_{i}}\right),\left(x_{n_{0}+\ldots+n_{j-1}+1}, \ldots, x_{n_{0}+\ldots+n_{j}}\right)$ существенных переменных любых двух функций $f_{i}$ и $f_{j}$ не пересекаются при $i \neq j$.

Утверждение 1.2. Если двоичная функция $f\left(x_{1}, \ldots, x_{n}\right)$ представима в виде конъюнкиии функций $\bigwedge_{i=1}^{t} f_{i}\left(x_{n_{0}+\ldots+n_{i-1}+1}, \ldots, x_{n_{0}+\ldots+n_{i}}\right), n_{0}=0$, от попарно не пересекающихся наборов переменных $\left(x_{n_{0}+\ldots+n_{i-1}+1}, \ldots, x_{n_{0}+\ldots+n_{i}}\right)$, $i=\overline{1, t}, u \sum_{i=1}^{t} n_{i} \leq n$, то коэффищиенты $\Delta_{\alpha}^{f}$ вычисляются по формулам

$$
\Delta_{\alpha}^{f}=\left\{\begin{array}{c}
2^{n-1}-2^{n-\sum_{i=1}^{t} n_{i}} \prod_{i=1}^{t}\left(2^{n_{i}-1}-\Delta_{\theta}^{f_{i}}\right), \text { если } \alpha=\theta, \\
(-1)^{h-1} 2^{n-\sum_{i=1}^{t} n_{i}} \prod_{j=1}^{h} \Delta_{\alpha_{i_{j}}}^{f_{i_{j}}} \prod_{j=h+1}^{t}\left(2^{n_{i_{j}}-1}-\Delta_{\theta}^{f_{i_{j}}}\right), \text { если } \alpha_{i_{1}} \neq \theta, \ldots, \\
\alpha_{i_{h}} \neq \theta, 1 \leq h \leq t, \alpha_{j}=\theta \text { nри } j \notin\left\{i_{1}, \ldots, i_{h}\right\} \text { u } \alpha_{0}=\theta, \\
(-1)^{t-1} 2^{n-\sum_{i=1}^{t} n_{i}} \prod_{i=1}^{t} \Delta_{\alpha_{i}}^{f_{i}}, \text { если } \alpha_{1} \neq \theta, \ldots, \alpha_{t} \neq \theta, \alpha_{0}=\theta, \\
0, \text { если } \alpha_{0} \neq \theta,
\end{array}\right.
$$

где $\alpha=\left(a_{1}, \ldots, a_{n}\right) \in V_{n}, \alpha_{i}=\left(a_{n_{0}+\ldots+n_{i-1}+1}, \ldots, a_{n_{0}+\ldots+n_{i}}\right) \in V_{n_{i}}, i=\frac{(3)}{1, t}$, $u \alpha_{0}=\left(a_{n_{0}+\ldots+n_{t}+1}, \ldots, a_{n}\right) \in V_{n-n_{1}-\ldots-n_{t}}$.

Доказательство утверждения 1.2 проводится методом математической индукции с использованием утверждения 1.1 . 
Отметим еще одно свойство коэффициентов $\Delta_{\alpha}^{f}$, которое понадобится при проведении дальнейших доказательств.

Утверждение 1.3. Если двоичная функияия $f\left(x_{1}, \ldots, x_{n}\right)$ представима в виде элементарной дизъюнкичии $x_{1}^{b_{1}} \vee \ldots \vee x_{n}^{b_{n}}$, то коэффициченть $\Delta_{\alpha}^{f}$ вычисляются по формулам

$$
\Delta_{\alpha}^{f}= \begin{cases}-2^{n-1}+1, & \text { если } \alpha=\theta, \\ (-1)^{a_{1}\left(b_{1}+1\right)+\ldots+a_{n}\left(b_{n}+1\right)}, & \text { если } \alpha \neq \theta .\end{cases}
$$

Доказательство представленной формулы проводится непосредственной проверкой.

Если $\alpha=\theta$, то $\Delta_{\theta}^{f}=2^{n-1}-\|f(x)\|=-2^{n-1}+1$.

Если $\alpha \neq \theta$, то достаточно воспользоваться формулой

$$
\Delta_{\alpha}^{f}=2\|f(x) \wedge(\alpha, x)\|-\|f(x)\|
$$

и условием

$$
x_{1}^{b_{1}} \vee \ldots \vee x_{n}^{b_{n}}=0 \Leftrightarrow\left(x_{1}, \ldots, x_{n}\right)=\left(b_{1} \oplus 1, \ldots, b_{n} \oplus 1\right)=\beta .
$$

В этом случае величина

$$
\|f(x) \wedge(\alpha, x)\|= \begin{cases}\|(\alpha, x)\|, & \text { если }(\alpha, \beta)=0 \\ \|(\alpha, x)\|-1, & \text { если }(\alpha, \beta)=1,\end{cases}
$$

и утверждение доказано.

\section{2. Вычисление коэффициента аддитивности для биюнктивных, слабо положительных и слабо отрицательных булевых функций частного вида}

Рассмотрим вопрос о величине коэффициента аддитивности биюнктивных, слабо положительных и слабо отрицательных функций. Будем считать, что функции представлены конъюнкцией функций от непересекающихся наборов переменных.

Рассмотрим КНФ функции $f\left(x_{1}, \ldots, x_{n}\right)$ вида

$$
f \equiv \bigwedge_{i=1}^{t} f_{i} \equiv \bigwedge_{i=1}^{t}\left(x_{s_{i, 1}}^{b_{i, 1}} \vee x_{s_{i, 2}}^{b_{i, 2}} \vee \ldots \vee x_{s_{i, n_{i}}}^{b_{i, n_{i}}}\right)
$$

где $b_{i, j} \in\{0,1\}, i=\overline{1, t}, j=\overline{1, n_{i}}, \sum_{i=1}^{t} n_{i} \leq n, n_{i} \geq 1$. 
Докажем утверждение, пользуясь которым, удобно вычислять коэффициент аддитивности булевой функции.

Утверждение 2.1. Пусть двоичная функичия $f\left(x_{1}, \ldots, x_{n}\right)$ представима в виде конъюнкиии $f \equiv \bigwedge_{i=1}^{t}\left(x_{s_{i, 1}}^{b_{i, 1}} \vee x_{s_{i, 2}}^{b_{i, 2}} \vee \ldots \vee x_{s_{i, n_{i}}}^{b_{i, n_{i}}}\right)$, а множества переменных $\left(x_{s_{i, 1}}, x_{s_{i, 2}}, \ldots, x_{s_{i, n_{i}}}\right), i \in \overline{1, t}$, попарно не пересекаются. Тогда

$$
\begin{array}{r}
\operatorname{Ad}(f)=\frac{1}{2}+\frac{1}{2^{3 \cdot \sum_{i=1}^{t} n_{i}-2}} \cdot\left(\left(2^{\sum_{i=1}^{t} n_{i}-1}-\prod_{i=1}^{t}\left(2^{n_{i}}-1\right)\right)^{3}-\right. \\
\left.-\sum_{h=1}^{t} \sum_{\substack{1 \leq i_{1}<\ldots<i_{h} \leq t, i_{h+1}, \ldots, i_{t} \in \overline{1, t} \backslash\left\{i_{1}, \ldots, i_{h}\right\}}}\left(\prod_{j=1}^{h}\left(1-2^{n_{i_{j}}} b_{i_{j}, 1} \cdot \ldots \cdot b_{i_{j}, n_{i_{j}}}\right) \cdot \prod_{j=h+1}^{t}\left(2^{n_{i_{j}}}-1\right)^{3}\right)\right) .
\end{array}
$$

Доказательство. Для вычисления коэффициента аддитивности функции воспользуемся формулой (2) и результатами утверждений 1.1-1.3. Будем считать, что вектор $\alpha=\left(a_{1}, \ldots, a_{n}\right)$ разбит на $t$ подвекторов $\alpha_{i}=$ $=\left(a_{s_{i, 1}}, a_{s_{i, 2}}, \ldots, a_{s_{i, n_{i}}}\right), i \in \overline{1, t}$, и вектор $\alpha_{0}$, содержащий координаты, не вошедшие в векторы $\alpha_{1}, \ldots, \alpha_{t}$.

Используя формулу, приведенную в утверждении 1.2, вычислим значение коэффициента $\Delta_{\alpha}^{f}$.

Предположим, что $\alpha=\theta$. В силу того что для каждого $i \in \overline{1, t}$ функция $f_{i}=x_{s_{i, 1}}^{b_{i, 1}} \vee x_{s_{i, 2}}^{b_{i, 2}} \vee \ldots \vee x_{s_{i, n_{i}}}^{b_{i, n_{i}}}$ существенно зависит от $n_{i}$ переменных и ее вес равен $2^{n_{i}}-1$, величина $\Delta_{\theta}^{f}$ вычисляется по формуле

$$
\Delta_{\theta}^{f}=2^{n-1}-2^{n-\sum_{i=1}^{t} n_{i}} \prod_{i=1}^{t}\left(2^{n_{i}}-1\right) .
$$

Пусть $\alpha \neq \theta$. Не ограничивая общности, будем считать, что векторы $\alpha_{j}=\left(a_{s_{j, 1}}, a_{s_{j, 2}}, \ldots, a_{s_{j, n_{j}}}\right), j \in \overline{1, h}, 1 \leq h \leq t$, ненулевые. Согласно утверждению 1.3 величина коэффициента $\Delta_{\alpha_{j}}^{f_{j}}$ для каждого $j \in \overline{1, h}$ вычисляется по формуле

$$
\Delta_{\alpha_{j}}^{f_{j}}=(-1)^{a_{s_{j, 1}}\left(b_{s_{j, 1}}+1\right)+\ldots+a_{s_{j, n_{j}}}\left(b_{s_{j, n_{j}}}+1\right)} .
$$

Применяя выражение (3), находим:

$\Delta_{\alpha}^{f}=-2^{n-\sum_{i=1}^{t} n_{i}} \cdot \prod_{j=1}^{h}\left((-1)^{a_{i_{j}, 1}\left(b_{i_{j}, 1}+1\right)+\ldots+a_{i_{j}, n_{i j}}\left(b_{i_{j}, n_{i j}}+1\right)+1}\right) \cdot \prod_{j=h+1}^{t}\left(2^{n_{i_{j}}}-1\right)$. 
Подставив эти формулы в выражение (2) и просуммировав результат по всем векторам $\alpha \in V_{n}$, получим доказываемое равенство.

Следствиями утверждения 2.1 являются выражения для коэффициентов аддитивности биюнктивных, слабо положительных и слабо отрицательных функций специального вида.

Следствие 2.1. Если двоичная функиия $f\left(x_{1}, \ldots, x_{n}\right)$ представима в виде конъюнкиии $f \equiv \underset{i=1}{\wedge}\left(x_{s_{i, 1}}^{b_{i, 1}} \vee x_{s_{i, 2}}^{b_{i, 2}}\right)$, а множества переменных $\left(x_{s_{i, 1}}, x_{s_{i, 2}}\right)$, $i \in \overline{1, t}$, попарно не пересекаются, то коэффициент аддитивности функции $f\left(x_{1}, \ldots, x_{n}\right)$ вычисляется по формуле

$$
\begin{gathered}
\operatorname{Ad}(f)=\frac{1}{2}+\frac{1}{2}\left(\left(1-2^{(1-2 t)} 3^{t}\right)^{3}-\left(2^{(1-2 t)} 3^{t}\right)^{3} .\right. \\
\left.\left(\sum_{h=1}^{t} 3^{-3 h} \sum_{1 \leq i_{1}<\ldots<i_{h} \leq t} \prod_{j=1}^{h}\left(1-4 b_{i_{j}, 1} b_{i_{j}, 2}\right)\right)\right) .
\end{gathered}
$$

Следствие 2.2. Если двоичная функичи $f\left(x_{1}, \ldots, x_{n}\right)$ представима в виде конъюнкиии $f \equiv \bigwedge_{i=1}^{t}\left(x_{s_{i, 1}}^{b_{i, 1}} \vee x_{s_{i, 2}} \vee \ldots \vee x_{s_{i, n_{i}}}\right)$, а множества переменных $\left(x_{s_{i, 1}}, x_{s_{i, 2}}, \ldots, x_{s_{i, n_{i}}}\right), \quad i \in \overline{1, t}$, попарно не пересекаются, то коэффициент аддитивности функиии $f\left(x_{1}, \ldots, x_{n}\right)$ вычисляется по формуле

$$
\begin{aligned}
& \operatorname{Ad}(f)=\frac{1}{2}+\frac{1}{2^{3 \cdot \sum_{i=1}^{t} n_{i}-2}}\left(\left(2^{\sum_{i=1}^{t} n_{i}-1}-\prod_{i=1}^{t}\left(2^{n_{i}}-1\right)\right)^{3}-\right. \\
& \left.-\sum_{h=1}^{t} \sum_{\substack{1 \leq i_{1}<\ldots<i_{h} \leq t, i_{h+1}, \ldots, i_{t} \in \overline{1, t} \backslash\left\{i_{1}, \ldots, i_{h}\right\}}}\left(\prod_{j=1}^{h}\left(1-2^{n_{i_{j}}} b_{i_{j}, 1}\right) \cdot \prod_{j=h+1}^{t}\left(2^{n_{i_{j}}}-1\right)^{3}\right)\right) .
\end{aligned}
$$

Следствие 2.3. Если двоичная функция $f\left(x_{1}, \ldots, x_{n}\right)$ представима в виде конъюнкиии $f \equiv \underset{i=1}{\wedge}\left(x_{s_{i, 1}}^{b_{i, 1}} \vee \bar{x}_{s_{i, 2}} \vee \ldots \vee \bar{x}_{s_{i, n_{i}}}\right)$, а множества переменных $\left(x_{s_{i, 1}}, x_{s_{i, 2}}, \ldots, x_{s_{i, n_{i}}}\right), i \in \overline{1, t}$, попарно не пересекаются, то коэффицичент 
аддитивности функиии $f\left(x_{1}, \ldots, x_{n}\right)$ вычисляется по формуле

$$
\begin{aligned}
& \operatorname{Ad}(f)=\frac{1}{2}+\frac{1}{2^{3 \cdot \sum_{i=1}^{t} n_{i}-2}}\left(\left(2^{\sum_{i=1}^{t} n_{i}-1}-\prod_{i=1}^{t}\left(2^{n_{i}}-1\right)\right)^{3}-\right. \\
& \left.-\sum_{h=1}^{t} \sum_{\substack{1 \leq i_{1}<\ldots<i_{h} \leq t, i_{h+1}, \ldots, i_{t} \in 1, t \backslash\left\{i_{1}, \ldots, i_{h}\right\}}}\left(\prod_{j=1}^{h}(-1)^{b_{i_{j}, 1} \cdot \delta_{n_{i_{j}}, 1}} \cdot \prod_{j=h+1}^{t}\left(2^{n_{i_{j}}}-1\right)^{3}\right)\right),
\end{aligned}
$$

где $\delta_{n, 1}=\left\{\begin{array}{l}0, \text { если } n \neq 1, \\ 1, \text { если } n=1 .\end{array}\right.$

Формулы (4)-(6) зависят от многих параметров, что затрудняет вывод качественных оценок величин коэффициентов аддитивности изучаемых классов функций в общем случае. В связи с этим приведем ряд примеров, демонстрирующих изменение величины коэффициента аддитивности при изменении параметров функции.

Рассмотрим множество функций, принадлежащих классам слабо положительных $(W P)$ и слабо отрицательных $(W N)$ функций. Положим $n=8$, $t=1,2,4, n_{i}=\frac{8}{t}$, где $i \in \overline{1, t}$. Числу $\beta=\sum_{i=0}^{t-1}\left(b_{i+1,1} \cdot 2^{t-i-1}\right)$ сопоставим двоичный вектор $\left(b_{1,1}, b_{2,1}, \ldots, b_{t, 1}\right)$.

Вычисленные величины коэффициентов аддитивности рассматриваемых функций запишем в таблицу. Условимся считать, что номера столбцов таблицы соответствуют числу $\beta$, а номера строк - параметру $t$. При этом каждая строка разбита на две подстроки: $W P$ и $W N$. Если число $i=$ $=\sum_{k=0}^{t-1}\left(i_{k+1,1} \cdot 2^{t-k-1}\right)$, то на пересечении $i$-го столбца и $j$-й строки таблицы в подстроке $W P$ приведено значение коэффициента аддитивности функции $f \equiv \stackrel{\wedge}{\wedge}\left(x_{s_{k, 1}}^{i_{k, 1}} \vee x_{s_{k, 2}} \vee \ldots \vee x_{s_{k, n_{k}}}\right)$, а в подстроке $W N-$ значение коэффициента аддитивности функции $f \equiv \underset{k=1}{\wedge}\left(x_{s_{k, 1}}^{i_{k, 1}} \vee \bar{x}_{s_{k, 2}} \vee \ldots \vee \bar{x}_{s_{k, n_{k}}}\right)$. Для вычисления коэффициента аддитивности использованы формулы (5) и (6).

Заметим, что с изменением параметра $t$ величина коэффициента аддитивности функции $f$ также изменяется. В частности, при $t=1$ значения величины $\operatorname{Ad}(f)$ близки к величине коэффициента аддитивности аффинной функции. А при $t=4$ коэффициенты аддитивности соответствующих функций принимают значения, наиболее близкие к значению коэффициента аддитивности бент-функции от $n=8$ переменных, равному $\frac{1}{2}+\frac{1}{2^{n-1}}$. 
Таблица 1. $n=8, t=1, n_{1}=8$

\begin{tabular}{|c|c|c|c|}
\hline \multicolumn{2}{|c|}{${ }_{t}>\beta$} & 0 & 1 \\
\hline \multirow{2}{*}{1} & $W P$ & $\frac{3 \cdot 127}{2^{15}} \approx 0,012$ & $\frac{383}{2^{15}} \approx 0,012$ \\
\hline & $W N$ & $\frac{3 \cdot 127}{2^{15}} \approx 0,012$ & $\frac{3 \cdot 127}{2^{15}} \approx 0,012$ \\
\hline
\end{tabular}

Таблица 2. $n=8, t=2, n_{1}=n_{2}=4$

\begin{tabular}{|c|c|c|c|c|c|}
\hline \multicolumn{2}{|c|}{$\beta$} & 0 & 1 & 2 & 3 \\
\hline \multirow{2}{*}{2} & $W P$ & $\frac{3 \cdot 3067}{2^{15}} \approx 0,281$ & $\frac{9623}{2^{15}} \approx 0,294$ & $\frac{9623}{2^{15}} \approx 0,294$ & $\frac{11^{2} \cdot 83}{2^{15}} \approx 0,306$ \\
\cline { 2 - 6 } & $W N$ & $\frac{3 \cdot 3067}{2^{15}} \approx 0,281$ & $\frac{3 \cdot 3067}{2^{15}} \approx 0,281$ & $\frac{3 \cdot 3067}{2^{15}} \approx 0,281$ & $\frac{3 \cdot 3067}{2^{15}} \approx 0,281$ \\
\hline
\end{tabular}

Таблица 3. $n=8, t=4, n_{1}=n_{2}=n_{3}=n_{4}=4$

\begin{tabular}{|c|c|c|c|c|c|}
\hline \multicolumn{2}{|c|}{$\beta$} & 0 & 1 & 2 & 3 \\
\hline \multirow{2}{*}{4} & $W P$ & $\frac{3 \cdot 5 \cdot 1103}{2^{15}} \approx 0,505$ & $\frac{17231}{2^{15}} \approx 0,526$ & $\frac{17231}{2^{15}} \approx 0,526$ & $\frac{103 \cdot 173}{2^{15}} \approx 0,544$ \\
\cline { 2 - 6 } & $W N$ & $\frac{3 \cdot 5 \cdot 1103}{2^{15}} \approx 0,505$ & $\frac{3 \cdot 5 \cdot 1103}{2^{15}} \approx 0,505$ & $\frac{3 \cdot 5 \cdot 1103}{2^{15}} \approx 0,505$ & $\frac{3 \cdot 5 \cdot 1103}{2^{15}} \approx 0,505$ \\
\hline
\end{tabular}

\begin{tabular}{|c|c|c|c|c|c|}
\hline \multicolumn{2}{|c|}{$t_{t} \quad \beta$} & 4 & 5 & 6 & 7 \\
\hline \multirow{2}{*}{4} & $W P$ & $\frac{17231}{2^{15}} \approx 0,526$ & $\frac{103 \cdot 173}{2^{15}} \approx 0,544$ & $\frac{103 \cdot 173}{2^{15}} \approx 0,544$ & $\frac{73 \cdot 251}{2^{15}} \approx 0,559$ \\
\hline & $W N$ & $\frac{3 \cdot 5 \cdot 1103}{2^{15}} \approx 0,505$ & $\frac{3 \cdot 5 \cdot 1103}{2^{15}} \approx 0,505$ & $\frac{3 \cdot 5 \cdot 1103}{2^{15}} \approx 0,505$ & $\frac{3 \cdot 5 \cdot 1103}{2^{15}} \approx 0,505$ \\
\hline
\end{tabular}

\begin{tabular}{|c|c|c|c|c|c|}
\hline \multicolumn{2}{|c|}{$\beta$} & 8 & 9 & 10 & 11 \\
\hline \multirow{2}{*}{4} & $W P$ & $\frac{17231}{2^{15}} \approx 0,526$ & $\frac{103 \cdot 173}{2^{15}} \approx 0,544$ & $\frac{103 \cdot 173}{2^{15}} \approx 0,544$ & $\frac{73 \cdot 251}{2^{15}} \approx 0,559$ \\
\cline { 2 - 6 } & $W N$ & $\frac{3 \cdot 5 \cdot 1103}{2^{15}} \approx 0,505$ & $\frac{3 \cdot 5 \cdot 1103}{2^{15}} \approx 0,505$ & $\frac{3 \cdot 5 \cdot 1103}{2^{15}} \approx 0,505$ & $\frac{3 \cdot 5 \cdot 1103}{2^{15}} \approx 0,505$ \\
\hline
\end{tabular}

\begin{tabular}{|c|c|c|c|c|c|}
\hline \multicolumn{2}{|c|}{$\beta$} & 12 & 13 & 14 & 15 \\
\hline \multirow{2}{*}{4} & $W P$ & $\frac{103 \cdot 173}{2^{15}} \approx 0,544$ & $\frac{73 \cdot 251}{2^{15}} \approx 0,559$ & $\frac{73 \cdot 251}{2^{15}} \approx 0,559$ & $\frac{5 \cdot 11^{2} \cdot 31}{2^{15}} \approx 0,572$ \\
\cline { 2 - 6 } & $W N$ & $\frac{3 \cdot 5 \cdot 1103}{2^{15}} \approx 0,505$ & $\frac{3 \cdot 5 \cdot 1103}{2^{15}} \approx 0,505$ & $\frac{3 \cdot 5 \cdot 1103}{2^{15}} \approx 0,505$ & $\frac{3 \cdot 5 \cdot 1103}{2^{15}} \approx 0,505$ \\
\hline
\end{tabular}


Для произвольной булевой функции $f$ из рассматриваемых классов вычислить величину коэффициента $\mathrm{Ad}(f)$ с помощью методов, описанных выше, не удается. Основная сложность заключается в том, что переменная с одним и тем же индексом может присутствовать сразу в нескольких функциях, участвующих в записи КНФ функции $f$. В этом случае не выполняются условия свойств 1.1 и 1.2, являющихся ключевыми при доказательстве утверждения 2.1. Однако можно предложить формальный алгоритм вычисления коэффициента аддитивности.

\section{3. Алгоритм вычисления коэффициента аддитивности булевой функции}

Приведем описание алгоритма вычисления коэффициента аддитивности произвольной булевой функции, заданной конъюнктивной нормальной формой.

Обозначим через $f\left(x_{1}, \ldots, x_{n}\right)_{i_{1}, \ldots, i_{k}}^{b_{i_{1}}, \ldots, b_{i_{k}}}$ подфункцию функции $f\left(x_{1}, \ldots, x_{n}\right)$, у которой переменная $x_{i_{j}}$ фиксирована значением константы $b_{i_{j}}$, где $i_{j} \in \overline{1, n}, j \in \overline{1, k}, b_{i_{j}} \in\{0,1\}$.

Для коэффициентов $\Delta_{\alpha}^{f}$ функции $f$ верно следующее утверждение.

Утверждение 3.1 (см. [2]). Пусть $f \in F_{n}-$ произвольная двоччная функция от п переменных. Тогда для любого целого $0 \leq k \leq n$ и произвольной фиксачии переменных $x_{i_{1}}, \ldots, x_{i_{k}}$ выполнено равенство

$$
\Delta_{\alpha}^{f}=\sum_{\beta \in V_{k}}(-1)^{\left(\beta, \alpha^{\prime \prime}\right)} \Delta_{\alpha^{\prime}}^{f_{i_{1}, \ldots, i_{k}}^{b_{i_{1}, \ldots, b_{i}}}},
$$

где $\alpha=\left(a_{1}, \ldots, a_{n}\right) \in V_{n}, \alpha^{\prime}=\left(a_{i_{k+1}}, \ldots, a_{i_{n}}\right) \in V_{n-k}, \alpha^{\prime \prime}=\left(a_{i_{1}}, \ldots, a_{i_{k}}\right) \in V_{k}$.

Воспользовавшись результатами утверждений 1.2 и 3.1, сформулируем и докажем утверждение о представлении коэффициентов $\Delta_{\alpha}^{f}$ произвольной двоичной функции $f$, заданной в виде конъюнкции функций.

Утверждение 3.2. Рассмотрим двоичную функциию $f\left(x_{1}, \ldots, x_{n}\right)$, представленную в виде конъюнкции функиий $\bigwedge_{i=1}^{t} f_{i}\left(x_{1}, \ldots, x_{n}\right)$, где каждая функциия $f_{i}$ существвенно зависит от переменных $x_{i, 1}, \ldots, x_{i, n_{i}}, i \in \overline{1, t}$. Пусть $\left\{x_{1}, \ldots, x_{k}\right\}=\bigcup_{i=1}^{t-1} \bigcup_{j=i+1}^{t}\left(\left\{x_{i, 1}, \ldots, x_{i, n_{i}}\right\} \cap\left\{x_{j, 1}, \ldots, x_{j, n_{j}}\right\}\right)-$ множество 2015, T. 6, № 3, C. 75-88 
всех переменных, сущчественных хотя бы для двух функиий $f_{i}, f_{j}, 1 \leq i<j \leq t$. Тогда коэффичиенты $\Delta_{\alpha}^{f}$ функиии $f\left(x_{1}, \ldots, x_{n}\right)$ вычисляются по формулам

$$
\Delta_{\alpha}^{f}=\left\{\begin{array}{l}
\sum_{\beta \in V_{k}}\left((-1)^{\left(\beta, \alpha^{\prime \prime}\right)}\left(2^{n-1}-2^{n-k-\sum_{i=1}^{t} n_{i}^{\prime}} \prod_{i=1}^{t}\left(2^{n_{i}-1}-\Delta_{\theta}^{f_{i}^{\prime}}\right)\right)\right), \\
\quad \text { если } \alpha^{\prime}=\theta, \alpha_{0}=\theta, \\
\sum_{\beta \in V_{k}}\left((-1)^{\left(\beta, \alpha^{\prime \prime}\right)}(-1)^{h-1} 2^{n-k-\sum_{i=1}^{t} n_{i}^{\prime}} \prod_{j=1}^{h} \Delta_{\alpha_{i_{j}}}^{f_{i_{j}}^{\prime}} \prod_{j=h+1}^{t}\left(2^{n_{i_{j}}^{\prime}-1}-\Delta_{\theta}^{f_{i_{j}}^{\prime}}\right)\right), \\
\quad \text { если } \alpha_{i_{1}} \neq \theta, \ldots, \alpha_{i_{h}} \neq \theta, 1 \leq h \leq t, \\
\alpha_{j}=\theta \text { при } j \notin\left\{i_{1}, \ldots, i_{h}\right\} \text { и } \alpha_{0}=\theta, \\
\sum_{\beta \in V_{k}}\left((-1)^{\left(\beta, \alpha^{\prime \prime}\right)}(-1)^{t-1} 2^{n-k-\sum_{i=1}^{t} n_{i}^{\prime}} \prod_{i=1}^{t} \Delta_{\alpha_{i}}^{f_{i}^{\prime}}\right) \\
\text { если } \alpha_{1}^{\prime} \neq \theta, \ldots, \alpha_{t}^{\prime} \neq \theta, \alpha_{0}=\theta,
\end{array}\right.
$$

где $f_{i}^{\prime}=f_{i}\left(x_{1}, \ldots, x_{n}\right)_{1, \ldots, k}^{b_{1}, \ldots, b_{k}}, n_{i}^{\prime}-$ число существенных переменньх функизии $f_{i}^{\prime}, \alpha=\left(a_{1}, \ldots, a_{n}\right), \alpha^{\prime \prime}=\left(a_{1}, \ldots, a_{k}\right), \alpha_{i}^{\prime}=\left(a_{i, 1}, \ldots, a_{i, n_{i}^{\prime}}\right), \alpha_{0}-$ вектор, состоящий из координат вектора $\alpha$, не вошедиих в вектор $\alpha^{\prime \prime}$ и векторы $\alpha_{1}^{\prime}, \ldots, \alpha_{t}^{\prime}$.

Для доказательства утверждения 3.2 достаточно заметить, что множества существенных переменных функций $f_{i}^{\prime}=f_{i}\left(x_{1}, \ldots, x_{n}\right)_{1, \ldots, k}^{b_{1}, \ldots, b_{k}}, i \in \overline{1, t}$, попарно не пересекаются. Следовательно, выполнены условия утверждения 1.2. Применяя утверждения 1.2 и 3.1, получаем искомую формулу для коэффициента $\Delta_{\alpha}^{f}$ произвольной двоичной функции $f\left(x_{1}, \ldots, x_{n}\right)$, заданной в виде КНФ.

Отметим, что трудоемкость вычисления коэффициентов $\Delta_{\alpha}^{f}$ по формуле, приведенной в утверждении 3.2, не превосходит сложности алгоритма быстрого преобразования Фурье (БПФ) нахождения коэффициентов $\Delta_{\alpha}^{f}$. В худшем случае, когда $k=n$ и все переменные встречаются не менее чем в двух функциях, сложность вычисления $\Delta_{\alpha}^{f}$ совпадает со сложностью БПФ, равной $O\left(n \cdot 2^{n}\right)$ операций поля, над которым задана рассматриваемая функция.

Приведем формальное описание алгоритма вычисления коэффициента аддитивности булевой функции, заданной в форме КНФ. 


\section{Вход алгоритма}

- КНФ функции $f \equiv \bigwedge_{i=1}^{t} f_{i}\left(x_{1}, \ldots, x_{n}\right) \in F_{n}$.

\section{IIaг 1}

- для каждой пары функций $\left(f_{i}, f_{j}\right), 1 \leq i<j \leq t$, определить пересечение множеств их существенных переменных $U_{i, j}=$ $=\left\{x_{i, 1}, \ldots, x_{i, n_{i}}\right\} \bigcap\left\{x_{j, 1}, \ldots, x_{j, n_{j}}\right\}$;

- вычислить $\bigcup_{i=1}^{t-1} \bigcup_{j=i+1}^{t} U_{i, j}=\left\{x_{h_{1}}, \ldots, x_{h_{k}}\right\}$.

\section{Шаг 2}

- для каждой функции $f_{i}^{\prime}=f_{i}\left(x_{1}, \ldots, x_{n}\right)_{h_{1}, \ldots, h_{k}}^{b_{h_{1}}, \ldots, b_{h_{k}}}$, существенно зависящей от $n_{i}^{\prime}$ переменных, $i \in \overline{1, t}$, вычислить коэффициенты $\Delta_{\alpha_{i}^{\prime}}^{f_{i}^{\prime}}$, $\alpha_{i}^{\prime}=\left(a_{i, 1}, \ldots, a_{i, n_{i}^{\prime}}\right) \in V_{n_{i}^{\prime}}$.

\section{Шаг 3}

- воспользовавшись результатами утверждения 3.2, определить значения $\Delta_{\alpha}^{f}$ для каждого вектора $\alpha \in V_{n}$.

\section{Шаг 4}

- подставляя значения коэффициентов $\Delta_{\alpha}^{f}$ в формулу (2), вычислить величину $\operatorname{Ad}(f)=\frac{1}{2}+\frac{1}{2^{3 n-2}} \sum_{\alpha \in V_{n}}\left(\Delta_{\alpha}^{f}\right)^{3}$.

\section{Выход алгоритма}

- значение коэффициента аддитивности функции $f$.

Представленным алгоритмом можно пользоваться для вычисления коэффициентов $\Delta_{\alpha}^{f}$ и коэффициента аддитивности произвольной двоичной функции $f$, принадлежащей классу биюнктивных, слабо положительных или слабо отрицательных двоичных функций. Причем, как было отмечено ранее, трудоемкость вычислений коэффициентов $\Delta_{\alpha}^{f}$ в этом случае не превосходит трудоемкости алгоритма быстрого преобразования Фурье, примененного к рассматриваемой функции. 


\section{Список литературы}

[1] Глухов М.М., Закревский П. Н., “О коэффициентах аддитивности и аффинности дискретных функций”, Дискретн. матем., 24:1 (2012), 30-47.

[2] Глухов М.М., Шишков А.Б., Математическая логика. Дискретные функиии. Теория алгоритмов, СПб.: Лань, 2012, 416 с.

[3] Горшков С. П., "Применение теории $N P$-полных задач для оценки сложности решения систем булевых уравнений”, Обозр. прикл. и промышл. матем., 2:3 (1995), 325-398.

[4] Тарасов А.В., “О свойствах функций, представленных в виде 2-КНФ», Дискретн. матем., 13:4 (2001), 99-115;

[5] Schaefer T., "Complexity of satisfiability problems", Proc. 10th Annu. ACM Symp. on Theory of Computing, 1978, 216-226. 